\title{
Another Nasty Effect of Opioids: Attenuating the Benefits of Motivational Interviewing in Fibromyalgia?
}
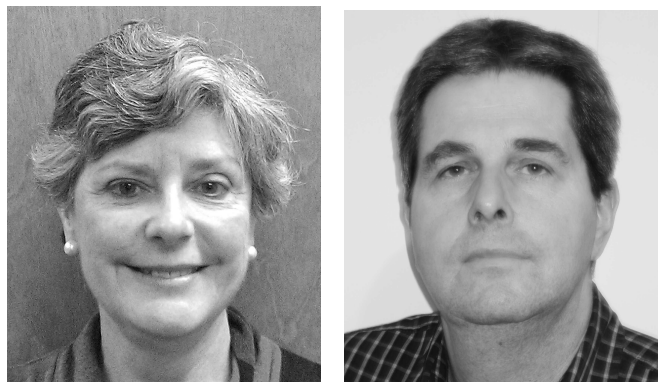

The reasons why fibromyalgia (FM) patients fail to adhere to medical advice are complex and multifactorial, with consequent challenge to effective medical care. While adherence to medication treatments is generally poor, adherence to lifestyle intervention recommendations may be even poorer $^{1,2}$. FM is a condition without cure at this time and with symptoms expected to be lifelong for most. Current guidelines recommend regular physical activity as a critical and necessary treatment strategy for FM; both short-term and longterm benefits of exercise have been demonstrated, emphasizing the need to promote adherence to these recommendations ${ }^{3,4,5,6}$. Nevertheless, continued practice of exercise by FM patients remains poor ${ }^{7}$. Strategies proposed to maintain adherence include patient education and empowerment, encouragement to participate in an activity that is pleasurable and convenient, and psychological interventions that may include cognitive behavioral training or motivational interviewing $(\mathrm{MI})^{8}$.

MI is a patient-centered technique that aims to facilitate patient involvement in maintaining good, health-centered behavior patterns. This psychological strategy, developed in the 1980 s, was initially used in the management of substance abuse disorders ${ }^{9}$. As the popularity of MI increased, along with the appreciation of the importance of active patient/person participation in health care, it was used in various other health-related settings such as prevention programs for smoking and alcohol use in youth, and then to a wider application to promote adherence to treatment recommendations in diverse diseases. The objective of MI is to sufficiently motivate a person to develop a closed loop of achievement, whereby a person is able to set and achieve goals that will continue to provide personal motivation by generating a self-sustaining loop.

MI may be delivered in various formats, including face-to-face encounter, telephone contact or online groups, as well as newer digital communication tools. The best method of providing MI has not been fully examined, but cost-saving methods, with a trend away from the face-to-face encounter, are increasingly reported. The success of MI can, however, be variable, with considerable evidence for positive effect in the first few months after the intervention, but often a less sustained effect in the long term. This has recently been demonstrated by findings of a metaanalysis of MI in the setting of pain management for adults with chronic pain. There was a small to moderate improvement in adherence to recommendations in the short term, but the effect was not sustained at followup ${ }^{10}$. However, prolonged intervention is costly, precluding longer duration of both intervention and study.

In an earlier study aimed to evaluate the efficacy of MI to promote exercise and improve symptoms of patients with FM, Ang and colleagues reported that MI was not successful in maintaining exercise activity by patients with FM at the 36-week followup ${ }^{11}$. In an effort to explore reasons for these negative results, the authors have performed a posthoc analysis of their original study by grouping patients according to opioid use, postulating that opioid use would counteract the benefits of $\mathrm{MI}^{12}$. The hypothesis is appealing in light of compelling evidence for negative effects of opioids in chronic pain conditions. Results of this analysis are reported in this issue of The Journal, and although they confirm the authors' hypothesis that opioid use is associated with poor adherence to continued exercise, we believe it is premature to entirely attribute this effect to adverse effects of opioids.

In the original large, single center study ${ }^{11}$, with a cohort of 216 participants, MI was administered by telephone on 6 occasions over a period of 12 weeks; and patients were then followed for 6 months. In line with previous reports, opioids were used by $33 \%$ of patients, with $25 \%$ of them using 2 or more classes of opioids. The patients participating in this study were, however, selected for more severe disease to avoid a ceiling effect of treatment and were therefore less representative of the usual FM population.

See Motivational interviewing for FM, page 505

Personal non-commercial use only. The Journal of Rheumatology Copyright @ 2017 . All rights reserved. 
The current study is a posthoc analysis of the original study; it has some limitations, some of which are acknowledged by the authors. Although use of opioids was recorded as "regularly", and not on an as-needed basis, there is no record of the reasons for opioid use, the doses patients consumed, their morphine-equivalent dose, and the duration of opioid treatment. Moreover, there are no data on possible discontinuation of opioid therapy during the study, or on commencing opioid therapy by nonusers. Patients were allocated to the opioid user/nonuser groups based on their report, but no confirming tests, such as urine drug screening, were done. Analyses were adjusted for the baseline value of outcome measures, which among others included an increase of at least $30 \mathrm{~min}$ of moderate to vigorous physical activity at the 6-month followup, as well as body mass index. However the opioid users versus nonusers differed on other characteristics that could have influenced the results. Users had significantly less education, higher depression scores, and greater disease severity as measured by the Fibromyalgia Impact Questionnaire, findings that have been reported by others $^{13}$.

Other disease-specific factors that may have influenced these results include duration of FM, severity of symptoms prior to opioid use, presence of other psychoactive drugs such as benzodiazepines, or substance use such as cannabis. Patients with FM do use a considerable amount of medication, emphasizing a tendency toward a chemical coping strategy. It is conceivable that those taking opioids had already received at least some of the recommended drug treatments, and opioids could have been chosen as a last resort. Patient-specific factors such as coping strategies, self-efficacy, and helplessness, personality characteristics such as catastrophizing or anxiety, and presence of kinesophobia could all also have played roles and influenced adherence to the same extent as the effect of opioids. Could it be that opioid users were sicker and therefore could not sustain the recommended physical activity?

Three additional factors regarding exercise should be considered as well. First, exercise routine before FM was diagnosed could influence illness-associated exercise. Those who have had a previous culture of regular physical activity may more easily appreciate the benefits of exercise and be willing to adhere to an exercise program. Second, about 30\% of patients with FM report that exercise induces more pain, with preliminary evidence that growth hormone dysfunction may be a factor ${ }^{14}$. Although the physiological basis for this complaint is not sufficiently understood, patients requiring more pain relief including opioids may have fallen into this category. Finally, although the opioid nonusers self-reported increased exercise activity, accelerometry-based physical activity did not differ for the MI versus the control group in the original study. This poor concordance between patient report of exercise activity and objective measurements has been reported for $\mathrm{FM}^{15}$. Therefore the validity of self-report of increased physical activity for the nonusers of opioid could be questioned.

Even with the above-mentioned limitations, the preliminary suggestion that opioids attenuate the beneficial effects of a standard psychological technique to improve treatment adherence is worrisome. While the common risks of opioid treatments are well known, Kim and colleagues ${ }^{12}$ raise an important, but likely unrecognized, adverse effect of opioid treatments: their possible effects on motivation. The effects of opioids on cognition may be subtle, especially when low doses are used, but may be sufficient to diminish interest in participation in activities ${ }^{16}$. Effects on cognition may also have reduced attention and memory required for retention of content at the time of the MI encounter. A second effect of opioids may be fatigue, which compounded with fatigue felt by many patients with FM, may preclude activity participation. Whether the absolute dose of opioids is critical to emergence of adverse effects is also unknown. It is possible that even a low dose of opioids suffices to affect motivation in susceptible persons and we know that adverse mood changes and hormonal effects are associated with more prolonged administration of opioids ${ }^{17,18}$. Finally, molecules closely aligned with opioids are the cannabinoids, which are known to induce an "amotivational" disorder ${ }^{19}$. Although it would be incorrect to extrapolate to opioids the cannabinoid effects on motivation, the similarities in adverse and psychoactive effects of these 2 drug categories suggests that amotivation may also be a plausible effect of opioids.

Further study is clearly needed before a negative blanket effect on psychological interventions, and MI in particular, can be attributed to opioid use. If opioids truly counteract the positive effect of MI in FM patients, are there important implications for continued application of a vast array of often costly psychological interventions in patients currently receiving opioid treatment? Although the study population experienced a distinct condition of chronic pain (FM), these effects, if valid, may be applicable to other chronic pain conditions as well. It is possible that Kim and colleagues have added another adverse effect to the mounting body of evidence against the use of opioids for management of chronic pain.

MARY-ANN FITZCHARLES, $\mathrm{MB}, \mathrm{Ch}$

Alan Edwards Pain Management Unit, McGill University Health Centre, and Division of Rheumatology, McGill University; YORAM SHIR, MD,

Alan Edwards Pain Management Unit, McGill University Health Centre, Montreal, Quebec, Canada.

Address correspondence to Dr. M.A. Fitzcharles, Montreal General Hospital, 1650 Cedar Ave., Montreal, Quebec H3G 1A4, Canada. E-mail: mary-ann.fitzcharles@muhc.mcgill.ca

Personal non-commercial use only. The Journal of Rheumatology Copyright @ 2017 . All rights reserved. 


\section{REFERENCES}

1. Sewitch MJ, Dobkin PL, Bernatsky S, Baron M, Starr M, Cohen M, et al. Medication non-adherence in women with fibromyalgia. Rheumatology 2004;43:648-54.

2. Dobkin PL, Ionescu-Ittu R, Abrahamowicz M, Baron M, Bernatsky S, Sita A. Predictors of adherence to an integrated multimodal program for fibromyalgia. J Rheumatol 2008;35:2255-64.

3. Fitzcharles MA, Ste-Marie PA, Goldenberg DL, Pereira JX, Abbey S, Choiniere M, et al. Canadian Pain Society and Canadian Rheumatology Association recommendations for rational care of persons with fibromyalgia. A summary report. J Rheumatol 2013;40:1388-93.

4. Ablin JN, Amital H, Ehrenfeld M, Aloush V, Elkayam O, Langevitz $\mathrm{P}$, et al. [Guidelines for the diagnosis and treatment of the fibromyalgia syndrome]. [Article in Hebrew]. Harefuah 2013;152:742-7, 750-1.

5. Eich W, Hauser W, Arnold B, Jackel W, Offenbacher M, Petzke F, et al. [Fibromyalgia syndrome. Definition, classification, clinical diagnosis and prognosis]. [Article in German] Schmerz 2012;26:247-58.

6. Macfarlane GJ, Kronisch C, Dean LE, Atzeni F, Hauser W, Fluss E, et al. EULAR revised recommendations for the management of fibromyalgia. Ann Rheum Dis 2017;76:318-28.

7. Hauser W, Klose P, Langhorst J, Moradi B, Steinbach M, Schiltenwolf M, et al. Efficacy of different types of aerobic exercise in fibromyalgia syndrome: a systematic review and meta-analysis of randomised controlled trials. Arthritis Res Ther 2010;12:R79.

8. Bernardy K, Klose P, Busch AJ, Choy EH, Hauser W. Cognitive behavioural therapies for fibromyalgia. Cochrane Database Syst Rev 2013;9:CD009796.

9. Miller WR. Motivational interviewing: research, practice, and puzzles. Addict Behav 1996;21:835-42.

10. Alperstein D, Sharpe L. The efficacy of motivational interviewing in adults with chronic pain: a meta-analysis and systematic review. J Pain 2016;17:393-403.

11. Ang DC, Kaleth AS, Bigatti S, Mazzuca SA, Jensen MP, Hilligoss J, et al. Research to encourage exercise for fibromyalgia (REEF): use of motivational interviewing, outcomes from a randomized-controlled trial. Clin J Pain 2013;29:296-304.

12. Kim S, Slaven JE, Ang DC. Sustained benefits of exercise-based motivational interviewing, but only among nonusers of opioids in patients with fibromyalgia. J Rheumatol 2017;44:505-11.

13. Fitzcharles MA, Faregh N, Ste-Marie PA, Shir Y. Opioid use in fibromyalgia is associated with negative health related measures in a prospective cohort study. Pain Res Treat 2013;2013:898493.

14. Ross RL, Jones KD, Bennett RM, Ward RL, Druker BJ, Wood LJ Preliminary evidence of increased pain and elevated cytokines in fibromyalgia patients with defective growth hormone response to exercise. Open Immunol J 2010;3:9-18.

15. Segura-Jimenez V, Alvarez-Gallardo IC, Romero-Zurita A, Camiletti-Moiron D, Munguia-Izquierdo D, Carbonell-Baeza A, et al. Comparison of physical activity using questionnaires (leisure time physical activity instrument and physical activity at home and work instrument) and accelerometry in fibromyalgia patients: the Al-Andalus project. Arch Phys Med Rehabil 2014;95:1903-11 e2.

16. Garland EL, Froeliger B, Zeidan F, Partin K, Howard MO. The downward spiral of chronic pain, prescription opioid misuse, and addiction: cognitive, affective, and neuropsychopharmacologic pathways. Neurosci Biobehav Rev 2013;37:2597-607.

17. Scherrer JF, Salas J, Copeland LA, Stock EM, Ahmedani BK, Sullivan MD, et al. Prescription opioid duration, dose, and increased risk of depression in 3 large patient populations. Ann Fam Med 2016;14:54-62.

18. Ali K, Raphael J, Khan S, Labib M, Duarte R. The effects of opioids on the endocrine system: an overview. Postgrad Med J 2016 Sep 19 (E-pub ahead of print).

19. Lawn W, Freeman TP, Pope RA, Joye A, Harvey L, Hindocha C, et al. Acute and chronic effects of cannabinoids on effort-related decision-making and reward learning: an evaluation of the cannabis 'amotivational' hypotheses. Psychopharmacology (Berl) 2016;233:3537-52.

J Rheumatol 2017;44:407-9; doi:10.3899/jrheum.170076 